\title{
Percepção sensorial e análise química de tempero e sal hipossódico como alternativas para dietas hipossódicas
}

Ana Carolina Bassi FORTES

Flávia DELLA LUCIA ${ }^{2 *}$

Pedro Orival LUCCAS ${ }^{3}$

Luciana AZEVEDO ${ }^{4}$

Eric Batista FERREIRA ${ }^{5}$

\begin{abstract}
${ }^{1}$ Farmacêutica pela Universidade Federal de Alfenas. E-mail: carolinabfortes@ hotmail.com
2* Professora do Departamento de Nutrição. Universidade Federal de Alfenas. E-mail: flavia@unifal-mg.edu.br. Autor para correspondência.

${ }^{3}$ Professor do Departamento de Ciências Exatas. Universidade Federal de Alfenas. E-mail: pedro@unifal-mg.edu.br ${ }^{4}$ Professora do Departamento de Nutrição. Universidade Federal de Alfenas. E-mail: lazevedo@unifal-mg.edu.br ${ }^{5}$ Professor do Instituto de Ciências Exatas. Universidade Federal de Alfenas. eric.ferreira@unifal-mg.edu.br.
\end{abstract}

\section{Recebido em: 22/08/2012 - Aprovado em: 10/12/2012 - Disponibilizado em: 30/12/2012}

RESUMO: O objetivo foi estudar a percepção sensorial do sal convencional (SC), do sal hipossódico (SH) e do tempero a base de sal hipossódico (TH) em arroz cozido relacionando- a com as quantidades de $\mathrm{Na}^{+}$e $\mathrm{K}^{+}$presentes nos mesmos. Foram preparadas amostras de arroz cozido com sal convencional, sal hipossódico e tempero a base de sal hipossódico em quatro concentrações $(3,00 \% ; 3,75 \% ; 4,50 \% ; 4,75 \%-\mathrm{m} / \mathrm{m})$. Determinou- se a umidade das amostras e as quantidades de sódio e potássio. As amostras de sal hipossódico não apresentaram boa aceitação. As concentrações de 3,75 e 4,50\% de tempero a base de sal hipossódico podem substituir o uso do sal convencional na sua concentração de melhor aceitação (3\%). Apesar da necessidade de maior acréscimo do TH para alcançar a mesma aceitação do convencional, ainda alcançou-se uma redução no consumo de sódio de aproximadamente $50 \%$.

Palavras-chave: sal hipossódico, tempero a base de sal hipossódico, sódio, potássio.

\begin{abstract}
Studying the sensorial perception of, conventional salt (CS), low sodium salt (LSS), and condiment with a low sodium salt base (CLSS) in cooked rice, relating it to the quantities of $\mathrm{NA}^{+}$and $\mathrm{K}^{+}$present within. Samples of cooked rice with CS, LSS and CLSS, were prepared in four concentrations $(3.00 \%, 3.75 \%, 4.50 \%$, and $4.50 \% \mathrm{~m} / \mathrm{m}$ raw rice). The moist of the cooked rice samples, sodium and potassium rates were determined. The samples of LSS didn't present a good acceptance. The concentrations of CLSS, $3.75 \%$ and $4.50 \%(\mathrm{~m} / \mathrm{m})$, may substitute the conventional salt in its best acceptance concentration $(3 \% \mathrm{~m} / \mathrm{m})$. Conclusion: Despite the necessity of a greater addition of CLSS, in order to reach the same level of acceptance as for CS, a reduction in the consumption of sodium of approximately $50 \%$ $(\mathrm{m} / \mathrm{m})$ could still be achieved.
\end{abstract}

Keywords: Low sodium salt, Condiment with a low sodium salt base, Sodium, Potassium.

\section{INTRODUÇÃO}

Sal é um nome genérico para uma família de substâncias com características químicas comuns, sendo que a mais importante, para o ser humano, é o cloreto de sódio $(\mathrm{NaCl})$ ou "sal de cozinha" ou "sal convencional". Esse, do ponto de vista nutricional, é fundamental para a saúde humana não apenas por ser utilizado de maneira universal no preparo e no processo de industrialização dos alimentos, mas também por ser consumido regularmente nas principais refeições do brasileiro (INMETRO, 2010). 
O sal convencional (SC) mostra-se um agente realçador do gosto e do sabor dos alimentos sendo, portanto, um fator interferente sobre as características sensoriais dos alimentos (BEN-SASSON, 2005). Mas, apesar de todos os benefícios sensoriais do cloreto de sódio, o seu excesso pode causar diversos prejuízos ao organismo, tais como a perda de cálcio urinário e a relação positiva com a incidência de câncer gástrico e hipertensão (VASQUEZ, 2005a).

A influência do $\mathrm{NaCl}$ na pressão arterial está relacionada com o histórico familiar de hipertensão arterial sistêmica e é intensificada com a idade, nos indivíduos normotensos. Evidencia-se que as doenças cardiovasculares constituem a principal causa de mortalidade no mundo e seu crescimento significativo nos países em desenvolvimento alerta para o potencial impacto nas classes menos favorecidas (RIQUE et al., 2002). A partir desse aspecto, as pesquisas têm mostrado que os consumidores estão buscando a redução da quantidade de sal em suas dietas (BRANDSMA, 2006).

Para essa finalidade, a indústria de alimentos disponibiliza no mercado consumidor substitutos do SC, onde parte do $\mathrm{NaCl}$ é substituído por cloreto de potássio $(\mathrm{KCl})$ e outros sais, como cloreto de amônio $\left(\mathrm{NH}_{4} \mathrm{Cl}\right)$. Entretanto, a maioria desses substitutos não consegue o sucesso desejado devido ao sabor residual amargo, causado especialmente pelo $\mathrm{KCl}$, utilizado como o principal sal substituto (VASQUEZ, 2005b).
Essas características desagradáveis impulsionaram a utilização de produtos para mascarar este gosto residual, dentre eles o ácido glutâmico (BEN-SASSON, 2005), sais de citrato, magnésio e cálcio (BONORDEN et al., 1998), fornecendo uma composição que fosse agradável ao paladar e que consequentemente, estimulasse sua utilização (VASQUEZ, 2005a).

Segundo a ANVISA (1995), o sal hipossódico (SH) é um produto elaborado a partir da mistura de cloreto de sódio com outros sais, de modo que a mistura final mantenha poder salgante semelhante ao do SC fornecendo, no máximo, $50 \%$ do teor de sódio na mesma quantidade de cloreto de sódio. Os ingredientes considerados obrigatórios na composição do sal hipossódico são o cloreto de sódio e de potássio e o iodo. A adição de "realçadores de sabor" no sal hipossódico, como por exemplo, o glutamato de sódio, glutamato de amônio, glutamato de cálcio, glutamato de potássio, fazem com que o produto final perca sua denominação de sal hipossódico e passe a ser classificado como um tempero a base de sal hipossódico (TH). Esses “realçadores de sabor" podem ser utilizados com o objetivo de mascarar o gosto residual do $\mathrm{KCl}$.

A proposta deste trabalho é estudar a percepção sensorial do sal convencional (SC), sal hipossódico ( $\mathrm{SH})$ e do tempero a base de sal hipossódico (TH) em preparações de arroz cozido, investigando a quantidade necessária 
para se obter um sabor mais aceitável sem afetar as condições de saúde. Pretendeu-se também avaliar a relação entre aceitação e consumo de $\mathrm{Na}^{+}$e $\mathrm{K}^{+}$, quantificado nas amostras por meio de análise química por fotometria de chama.

\section{MATERIAL E MÉTODOS}

\section{Amostras}

O método de preparo do arroz cozido foi realizado segundo Araújo (1995), com modificações. Para cada amostra adicionouse, em panela de alumínio, 150 gramas de arroz polido tipo $1,350 \mathrm{~mL}$ de $\mathrm{H}_{2} 0,6 \mathrm{~mL}$ de óleo de soja, além do produto salgante (sal convencional, sal hipossódico ou tempero a base de sal hipossódico) nas concentrações de $3,00 \% ; 3,75 \% ; 4,50 \% ; 4,75 \%(\mathrm{~m} / \mathrm{m}$ arroz crú).

A escolha das quantidades de salgantes utilizados foi baseada em estudos preliminares do grupo de pesquisa, que determinaram como a amostra de melhor aceitação para o SC a concentração de 3,00\%. As amostras de TH em concentrações inferiores a esse valor de melhor aceitação $(3,00 \%)$ não obtiveram bons resultados. Partindo destes dados, testes-piloto foram realizados a fim de se obter concentrações superiores a 3,00\% de SH e TH que tivessem aceitação semelhante às amostras de 3,00\% de SC.

\section{Análises Físico-Químicas}

A umidade das amostras foi determinada conforme metodologia proposta pela AOAC (1997), em estufa a $105^{\circ} \mathrm{C}$ até peso constante. A determinação do teor de sódio e potássio nas amostras de arroz cozido foi realizada por meio de fotometria de chama de acordo com as determinações da EMBRAPA (1997). Para a validação do método de determinação utilizou-se três parâmetros analíticos: linearidade, limite de detecção (LD) e limite de quantificação (LQ).

Todo o material utilizado durante a análise química das amostras foi descontaminado com ácido nítrico $10 \%$ por 24h e lavado com água milli-Q. Para a elaboração dos padrões, adotaram-se as concentrações de 0,$25 ; 0,50 ; 0,75 ; 1,00 ; 3,00$; 5,$00 ; 7,00$ e $10,00 \mathrm{mgL}^{-1}$, utilizando-se cloreto de sódio PA (MERCK®) e cloreto de potássio PA (MERCK®). As análises dos eletrólitos foram realizadas utilizando-se a massa seca das amostras de arroz cozido com os respectivos salgantes. Assim, uma massa de 0,5 g de arroz cozido seco, pesada em triplicata, foi digerida, em bloco digestor (MARCONI ${ }^{\circ}$ ) com temperatura de $130^{\circ} \mathrm{C}$, com 5mL ácido nítrico 70\% PA.

Após a digestão, as amostras foram colocadas em balão volumétrico de $50 \mathrm{~mL}$, completando-se o volume com água milli-Q e, sequencialmente, diluída (1mL:100mL água milli-Q). Fez-se, então, a leitura dos sinais de sódio e potássio em fotômetro de chama 
(MICRONAL®). O valor final foi determinado pela média dos valores convertidos em $\mathrm{g} \mathrm{Na}^{+}$e $\mathrm{K}^{+} \cdot 100 \mathrm{~g}^{-1}$ de arroz cozido. Os resultados foram analisados por meio de análise de variância (ANAVA) a 5\% de significância, seguida de análise de regressão.

\section{Análise Sensorial}

O teste de aceitação, com escala hedônica estruturada de nove pontos (extremos desgostei muitíssimo e gostei muitíssimo) (MINIM et al., 2003), foi conduzido com 63 consumidores potenciais dos produtos, não treinados, escolhidos ao acaso e com idade entre 18 e 60 anos. Foram esclarecidos de forma completa e em linguagem leiga a respeito do protocolo de pesquisa. O projeto obteve aprovação do Comitê de Ética em Pesquisa envolvendo seres humanos da Universidade Federal de Alfenas sob o protocolo de pesquisa 23087.001266/2008-28.

As sessões foram realizadas em cabines individuais no Laboratório de Análise Sensorial do Departamento de Nutrição da Universidade Federal de Alfenas. As amostras foram apresentadas de forma monádica e aleatória, em pires de fundo transparente, codificados com número de três dígitos (VIANNA, 1995). Foi orientado ao provador para que enxaguasse a boca com água antes do ínicio da análise e entre as amostras.
O delineamento utilizado foi o inteiramente casualizado (DIC) e a análise dos resultados foi por meio de análise de variância (ANAVA) a 5\% de significância, seguida de comparação de médias pelo teste de Tukey.

Todas as análises foram feitas no software R (R DEVELOPMENT CORE TEAM, 2011), por meio do pacote ExpDes (FERREIRA et al., 2011).

\section{RESULTADOS E DISCUSSÃO}

Segundo Vasquez (2005b), a razão pela qual a maioria dos substitutos do sal convencional não ter alcançado o sucesso desejado é causada pela presença, principalmente do $\mathrm{KCl}$, que confere um residual amargo e influencia no seu consumo. A presença desse flavor desagradável torna-se preocupante à medida que pode aumentar o consumo desse produto pelo consumidor, que busca um "tempero" agradável e similar ao paladar salgado a que está habituado. Nesse caso, a ingestão aumentada desse produto, mesmo com níveis diminuídos de sódio, pode fazer com que o consumidor tenha um consumo igual ou até mesmo maior desse eletrólito, comparativamente ao sal convencional. O presente estudo analisou as escolhas dos consumidores quanto aos três tipos de salgantes adicionados durante o cozimento do arroz, em quatro concentrações.

Nos resultados obtidos pela análise sensorial, observou-se diferença significativa 
$(\mathrm{p}<0,05)$ entre as amostras em relação à aceitação. Realizando-se o Teste de Tukey (Tabela 1), constatou-se que a amostra 3\% SC foi a que obteve a maior aceitação $(7,16$ entre os termos hedônicos "gostei moderadamente" e "gostei muito") e diferiu significativamente das amostras de $\mathrm{SH}$ $(3,00 \%, 3,75 \%, 4,50 \%, 4,75 \%)$ e de $\mathrm{TH}$
$(3,00 \%$ e $4,75 \%)$, as quais se situaram entre os termos hedônicos "gostei ligeiramente" e "indiferente". Ao contrário, não houve diferença entre as amostras de SC (3\%, $3,75 \%, 4,5 \%$ e $4,75 \%)$ e as de $\mathrm{TH}(3,75 \%$, $4,50 \%$ ), as quais se situaram entre os termos "gostei ligeiramente" e "gostei moderadamente" $(p>0,05)$.

Tabela 1 - Médias dos níveis de aceitação de amostras de arroz cozido com diferentes níveis de sal convencional (SC); sal hipossódico (SH) e tempero a base de sal hipossódico (TH).

\begin{tabular}{cc}
\hline Amostras & Médias de Aceitação $^{1}$ \\
\hline $3,00 \%$ SC & $7,16^{\mathrm{a}}$ \\
$4,50 \% \mathrm{TH}$ & $6,98^{\mathrm{ab}}$ \\
$4,75 \% \mathrm{SC}$ & $6,91^{\mathrm{abc}}$ \\
$4,50 \% \mathrm{SC}$ & $6,83^{\mathrm{abc}}$ \\
$3,75 \% \mathrm{TH}$ & $6,62^{\mathrm{abcd}}$ \\
$3,75 \% \mathrm{SC}$ & $6,51^{\text {abcde }}$ \\
$3,00 \% \mathrm{SH}$ & $6,25^{\text {bcde }}$ \\
$4,75 \% \mathrm{TH}$ & $6,19^{\text {bcde }}$ \\
$4,50 \% \mathrm{SH}$ & $6,16^{\text {cde }}$ \\
$4,75 \% \mathrm{SH}$ & $6,14^{\text {cde }}$ \\
$3,00 \% \mathrm{TH}$ & 6,02 de \\
$3,75 \% \mathrm{SH}$ & $5,76{ }^{\mathrm{e}}$ \\
\hline Tédias seguidas pela mesma letra na coluna não diferem estatisticamente entre si pelo teste de Tukey (p>0,05).
\end{tabular}

Nota-se que o $\mathrm{TH}$ somente atingiu aceitação semelhante à amostra de 3,00\% SC, considerada como a de melhor aceitação pelos provadores, quando se aumenta as concentrações desse tempero para 3,75 e 4,50\% como evidenciado na Figura 1.

Para o $\mathrm{SH}$, a amostra $3,75 \%$ obteve a menor aceitação $(5,76)$ situando-se entre os termos hedônicos "indiferente" e "gostei ligeiramente", porém não difere das amostras SC $(3,75 \%), \mathrm{SH}(3 \%, 3,75 \%, 4,5 \%, 4,75 \%) \mathrm{e}$ $\mathrm{TH}(4,75 \%)$. Nota-se que as amostras de $\mathrm{SH}$ obtiveram menor aceitação, causada provavelmente pelo residual amargo provocado pelo $\mathrm{KCl}$, embora níveis menores de $\mathrm{Na}^{+}$sejam encontrados nesse produto. Outros estudos encontraram diferenças na percepção sensorial deste produto. Ben- 
Sasson (2005) encontrou uma pequena diferença significativa em relação à aceitação de duas amostras de suco de tomate, uma adicionada de sal convencional e a outra de sal hipossódico.

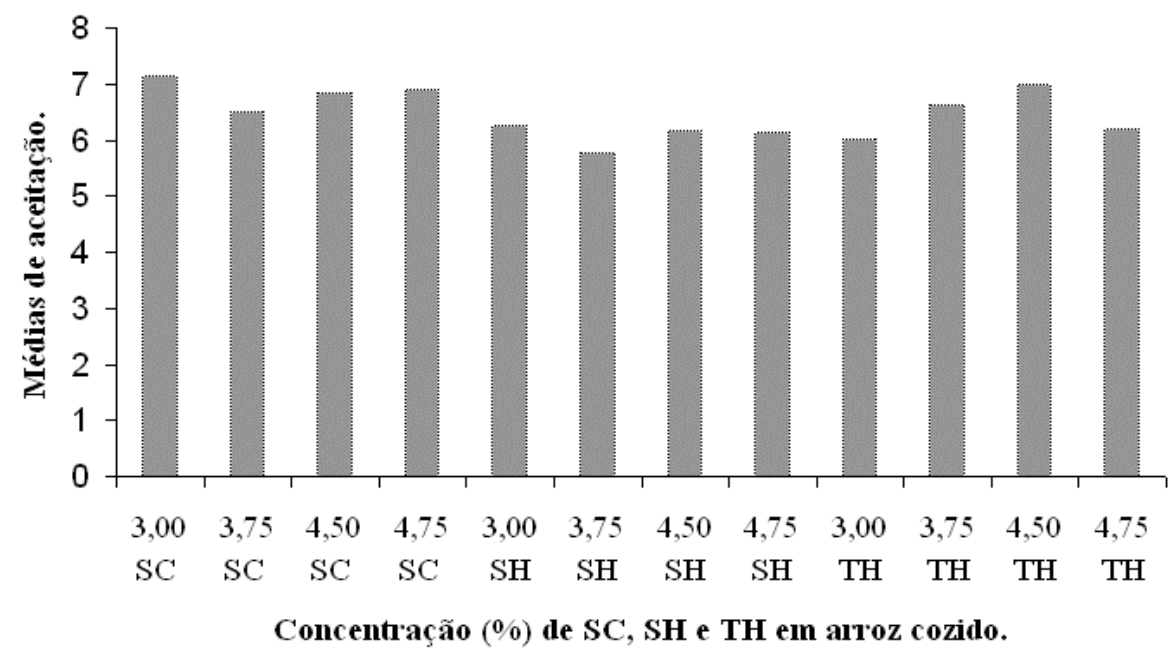

Figura 1 - Perfil de aceitação das amostras de arroz cozido com diferentes níveis de sal convencional (SC); sal hipossódico (SH) e tempero a base de sal hipossódico (TH).

Em consideração aos valores de escores médios, segundo Konkell et al. (2004), médias de aceitação na escala hedônica superiores a 6 demonstram valores consideráveis de aceitação do produto analisado frente aos consumidores. A única amostra que apresentou média inferior a 6 foi 3,75\% SH (média 5,76), justificando assim a substituição do SC por SH com aceitação agradável e níveis de $\mathrm{Na}^{+}$reduzidos em mais de $40 \%$.

Quanto às análises físico-químicas, pretendeu-se caracterizar as amostras oferecidas aos provadores visando a padronização e homogeneidade do preparo do arroz com o salgante, além de avaliar as quantidades de sódio e potássio frente à percepção sensorial. Segundo a Tabela Brasileira de Composição de Alimentos TACO (2006), a umidade encontrada em $100 \mathrm{~g}$ de arroz tipo1 cozido é de 69,1\%, confirmando que as condições de preparo resultaram em um teor de umidade semelhante ao processo utilizado pelos consumidores (Tabela 2).

A validação de um método analítico é o processo que tem por objetivo estabelecer que suas características de eficiência correspondam aos requerimentos necessários à aplicação analítica desejada (SIQUEIRAMOURA et al., 2008). Para a validação das análises dos íons, o método analítico utilizado mostrou-se linear no intervalo de 0,25 a 10 $\mathrm{mg}, \mathrm{L}^{-1}$, tanto para $\mathrm{Na}^{+}$quanto para $\mathrm{K}^{+}$, sendo 
que o coeficiente de correlação (r) foi respectivamente de 0,997 e 0,999 , compatível com a resolução RE $n^{\circ} 899$ da ANVISA, de 29 de maio de 2003 (BRASIL, 2003). O limite de detecção $\left(0,01 \mathrm{mgL}^{-1}\right)$ não diferiu de acordo com o íon, e o limite de quantificação foi de $0,03 \mathrm{mgL}^{-1}\left(\mathrm{Na}^{+}\right)$e $0,03 \mathrm{mgL}^{-1}\left(\mathrm{~K}^{+}\right)$.
Segundo a ANVISA (BRASIL, 1995), o limite de detecção é a menor quantidade do analito presente em uma amostra que pode ser detectado, porém não necessariamente quantificado.

Tabela 2 - Caracterização de umidade, $\mathrm{Na}^{+}$e $\mathrm{K}^{+}$das amostras de arroz com diferentes níveis de sal convencional (SC), sal hipossódico (SH) e tempero a base de sal hipossódico (TH).

\begin{tabular}{cccccc}
\hline Amostra* & \% UMIDADE & $\begin{array}{c}\text { Qde sal/ } \\
\text { arroz cru } \\
\left(\mathrm{g} .100 \mathrm{~g}^{-1}\right)\end{array}$ & $\begin{array}{c}\mathrm{Qde} \mathrm{Na}^{+} / \\
\text {arroz cozido } \\
\left(\mathrm{g} .100 \mathrm{~g}^{-1}\right)\end{array}$ & $\begin{array}{c}\mathrm{Qde} \mathrm{K}^{+} / \\
\text {arroz cozido } \\
\left(\mathrm{g} .100 \mathrm{~g}^{-1}\right)\end{array}$ & $\begin{array}{c}\% \text { de } \mathrm{Na}^{+} \text {em } \\
\text { relação à } \\
\text { amostra de } 3 \% \\
\mathrm{SC}(\mathrm{m} / \mathrm{m})\end{array}$ \\
\hline $3,00 \% \mathrm{SC}$ & $65 \pm 0,3$ & 3,01 & $1,17 \pm 0,01$ & - & $100 \%$ \\
$3,75 \% \mathrm{SC}$ & $61 \pm 0,2$ & 3,75 & $1,75 \pm 0,30$ & - & $149 \%$ \\
$4,50 \% \mathrm{SC}$ & $63 \pm 0,5$ & 4,50 & $1,77 \pm 0,06$ & - & $151 \%$ \\
$4,75 \% \mathrm{SC}$ & $64 \pm 2$ & 4,75 & $1,82 \pm 0,13$ & - & $155 \%$ \\
$3,00 \% \mathrm{SH}$ & $65 \pm 1,5$ & 3,01 & $0,53 \pm 0,11$ & $0,57 \pm 0,13$ & $45 \%$ \\
$3,75 \% \mathrm{SH}$ & $67 \pm 1,9$ & 3,75 & $0,67 \pm 0,04$ & $0,59 \pm 0,08$ & $57 \%$ \\
$4,50 \% \mathrm{SH}$ & $66 \pm 2,6$ & 4,50 & $0,70 \pm 0,19$ & $0,87 \pm 0,05$ & $60 \%$ \\
$4,75 \% \mathrm{SH}$ & $66 \pm 1,8$ & 4,75 & $0,90 \pm 0,05$ & $0,95 \pm 0,04$ & $77 \%$ \\
$3,00 \% \mathrm{TH}$ & $66 \pm 0,4$ & 3,00 & $0,41 \pm 0,06$ & $0,53 \pm 0,07$ & $35 \%$ \\
$3,75 \% \mathrm{TH}$ & $66 \pm 0,6$ & 3,75 & $0,50 \pm 0,12$ & $0,60 \pm 0,12$ & $42 \%$ \\
$4,50 \% \mathrm{TH}$ & $66 \pm 0,9$ & 4,51 & $0,68 \pm 0,07$ & $0,79 \pm 0,08$ & $58 \%$ \\
$4,75 \% \mathrm{TH}$ & $65 \pm 0,1$ & 4,75 & $0,74 \pm 0,06$ & $0,83 \pm 0,07$ & $64 \%$ \\
\hline
\end{tabular}

SC: sal convencional; SH: sal hipossódico; TH: tempero a base de sal hipossódico.

* Arroz cozido adicionado de diferentes percentuais de SC, SH, TH (g salgante/ g arroz cru).

${ }^{1}$ Quantidades obtidas por análise em fotometria de chama.

Já o limite de quantificação é a menor quantidade desse analito que pode ser determinada com precisão e exatidão aceitáveis. De acordo com os resultados, observou-se que a curva analítica apresentou como primeiro calibrador $\left(0,25 \mathrm{mgL}^{-1}\right)$ a concentração maior do que o LQ e o LD do método, o que também foi compatível com os critérios de validação preconizados (BRASIL, 2003).

Para os resultados das análises dos íons, observou-se diferença significativa 
$(p<0,05)$ entre as amostras em relação à concentração do salgante por meio da análise de variância. A análise de regressão demonstrou que o aumento da concentração do salgante repercutiu no aumento dos níveis de $\mathrm{Na}^{+}$e $\mathrm{K}^{+}$, independente do tipo de sal (Tabela 2). Esse aspecto foi coerente com o que se pretendia no preparo dessas amostras, ou seja, o emprego de salgantes em níveis crescentes e adequado preparo/cozimento, representaram níveis crescentes dos íons.

\section{CONCLUSÃO}

A partir dos resultados sensoriais e analíticos observou-se que as concentrações de 3,75 e 4,50\% TH podem substituir o uso do sal convencional na concentração de $3,00 \%$ SC sem alterações na aceitação e com redução de quase $50 \%$ nos níveis de sódio. Segundo Costa e Silva (2005) a dieta hipossódica induz à queda da pressão arterial por meio do aumento da natriurese, diminuição da secreção de renina e norepinefrina e aumento de secreção de prostaglandinas. Sendo assim, as amostras com melhor aceitação de TH poderão ser usadas em substituição ao SC, sem, contudo afetar a aceitação do produto. Vale salientar que o consumo de TH é contra-indicado em casos de uso de medicamentos poupadores de potássio e em pacientes com insuficiência renal crônica (MARTINS et al., 2003).

\section{AGRADECIMENTOS}

Os autores agradecem a Universidade Federal de Alfenas, aos provadores, à FAPEMIG pelo apoio financeiro e ao $\mathrm{CNPq}$ pela bolsa de IC concedida para Ana Carolina Bassi Fortes.

\section{REFERÊNCIAS BIBLIOGRÁFICAS}

ARAÚJO, M. O. D. Alimentos "Per Capita". $2^{a}$. Ed. Natal: Livraria Universitária; 1995.

ASSOCIATION OF OFFICIAL ANALYTICAL CHEMISTRY. Official Methods of Methods of Analysis of AOAC. Gaithersburg: AOAC, 1997.

BEN-SASSON, S. A., inventor; World Intellectual Property, cessionário. Salt substitute comprising biocompatible polymer. United States patent WO2005086566. 2005 Sep 22.

BONORDEN, W. R., inventor; GIORDANO, D. A., inventor; LEE, B. L., inventor; LUKIS, H. M., inventor; World Intellectual Property, cessionário. Salt flavor enhancing compositions. United States patent WO1998053708. 1998 Dez 03.

BRANDSMA, I. Reducing sodium: A European perspective. Food Technology 60(3):24-29. 2006. Acesso: 05 fev 2010. Disponível em: www.ift.org.

BRASIL. Agência Nacional de Vigilância Sanitária. Portaria $\mathrm{n}^{\circ}$ 54, de 04 de julho de 1995. Acesso em: 05 jan 2010. Disponível em: www.anvisa.gov.br. 
BRASIL. Agência Nacional de Vigilância Sanitária. Resolução - RE 822, de 29 de maio de 2003. Acesso em: 10 fev 2010. Disponível em: www.anvisa.gov.br.

COSTA, R. P.; SILVA, C. C. Doenças Cardiovasculares. Barueri: Livraria Manole; 2005.

FERREIRA, E. B.; CAVALCANTI, P. P.; NOGUEIRA, D. A. Experimental Designs: um pacote $\mathrm{R}$ para análise de experimentos. Revista da Estatística da UFOP, v. 1, n. 1, p. 1-9. 2011.

INMETRO. Instituto Nacional de Metrologia. Normalização e qualidade industrial,. Sal refinado. Brasil, 2010. Disponível em: http://www.inmetro.gov.br/consumidor/produ tos/sal2.asp.

KONKELL, F. E.; OLIVEIRA, S. M. R.; SIMÕES, D. R. S.; DEMIATE, I. M. Avaliação sensorial de doce de leite pastoso com diferentes concentrações de amido. Ciênc Tecnol Aliment. 2004, 24(2):249-54. doi: 10.1590/S0101-20612004000200015.

MARTINS, C.; MOREIRA, S. M.; PIEROSAN, S. R. Interações Droganutriente. Curitiba: Livraria Nutroclínica; 2003.

MINIM, V. P. R.; MACHADO, P. T.; CANAVESI, E.; PIROZI, M. R. Perfil sensorial e aceitabilidade de diferentes formulações de pão de queijo. Rev Nutr. 2003; 19(7):154-59. doi: 10.1590/S010120612000000200005.

R DEVELOPMENT CORE TEAM. $R: A$ language and environment for statistical computing. R Foundation for Statistical Computing, Vienna, Austria. 2011.
REAL, V. J.; FERRAZ, L. F. M.; RABELLO, L. M. Cuidados básicos com fotometria de chama. Embrapa. Acesso em: 05 jan 2010. Disponível em: www.embrapa.gov.br.

RIQUE, A. B. R.; SOARES, E. A.; MEIRELLES, C. M. Nutrição e exercício na prevenção e controle das doenças cardiovasculares. Rev Bras Med Esp. 2002; 8(6): 244-54. doi: 10.1590/S151786922002000600006 .

SIQUEIRA-MOURA, M. P.; LIRA, M. C. B.; SANTOS-MAGALHÃES, N. S. Validação de método analítico espectrofotométrico UV para determinação de ácido úsnico em lipossomas. Rev Bras Cienc Farm, 2008; 44(4):621-28. doi: 10.1590/S151693322008000400008 .

TABELA BRASILEIRA DE COMPOSIÇÃO DE ALIMENTOS. Núcleo de Estudos e Pesquisa em alimentação. Universidade Estadual de Campinas, Campinas: UNICAMP, 2006.

VASQUEZ, R. E. L., inventor; World Intellectual Property, cessionário. Non-bitter sodium-free or low-sodium salt composition. United States patent WO2005094615. 2005a Oct 13.

VASQUEZ, R. E., inventor; World Intellectual Property, cessionário. Stable sodium-free or low-sodium aqueous solution of agreeable saltiness taste. United States patent WO2005056477. 2005b Jun 23.

VIANNA, V. A. Influência da calda na qualidade de pêssego tipo passa. (dissertação). Universidade Federal de Pelotas; 1995. 